\title{
Short-term response of riparian vegetation to 4 grazing treatments
}

\author{
CARLO A. POPOLIZIO, HAROLD GOETZ, AND PHILLIP L. CHAPMAN
}

\begin{abstract}
Authors are research associate, Center for Ecological Management of Military Lands (CEMML). Department of Rangeland Ecosystem Science Department, Colorado State University, Fort Collins 80523; department head, Department of Rangeland Ecosystem Science, Colorado State University, Fort Collins 80523; and assistant professor, Department of Statistics, Colorado State University, Fort Collins 80523.
\end{abstract}

\begin{abstract}
The Sheep Creek watershed of northcentral Colorado provided an ideal site to collect baseline trend data and to estimate foliar cover responses of montane riparian vegetation. Percent relative

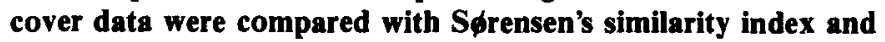
were analyzed with a 2-stage nested analysis of variance (ANOVA) to assess differences among 4 grazing treatments: long-term grazing (G), protection from livestock grazing since $1956(P)$, recent protection following long-term grazing (P88), and recent livestock grazing following protection (G88). This study utilized 3 replications of each treatment. Data were collected in August 1988, June 1989, and August 1989, employing permanent and randomly placed transects and plots.

When percent foliar cover means were paired using S $\phi$ rensen's similarity index, long-term grazing and short-term grazing treatments were least similar in August 1988. Long-term protection and short-term grazing were most similar in June 1989.

Average percent cover of bare ground, common dandelion (Taraxacum officinale Wiggers), white Dutch clover (Trifolium repens $\mathbf{L}$.), and legumes grouped as lifeforms were significantly different among treatments, with long-term grazing being significantly different from long-term protection. Average sedge and forb cover was least affected. However, responses of individual sedge species varied with treatments.

Average percent grass cover increased under short-term protection after a history of long-term grazing. Short-term grazing stimulated foliar cover of forbs, grasses, and sedges after more than 30 years of cattle exclusion.
\end{abstract}

Key Words: baseline trend, foliar cover, grazing, plant inventory, protection, riparian

Riparian ecosystems of the western United States were heavily impacted by trapping, road construction, logging, mining, agricultural conversions, grazing by domestic animals, water use, and recreational developments over approximately the past 140 years (Platts 1981, Skinner 1986, Knopf et al. 1988). Vegetation, soils, stream channel morphology, and water quality, as well as fish, wildlife, and invertebrate populations are greatly affected by these uses (Orr 1960, Laycock and Conrad 1967, Van Velson 1979, Platts and Raleigh 1984, Bohn and Buckhouse 1985, Platts and Nelson 1985, Knopf et al. 1988).

Multiple use management strategies implemented during the 1970 's highlighted in the poor condition of riparian corridors and provided opportunities for research on dynamics and characteris-

\footnotetext{
Research was funded by the Colorado Agricultural Experiment Station, through project 156502 and the USDA Rangeland Research Grants Program 532110. Mrs. Patricia Douglas, CEMML, and Mrs. Terry Tucker Schulz are gratefully acknowledged for support of the senior author during research and preparation of this manuscript.

Manuscript accepted 16 Aug. 1993.
}

tics of these systems. Many problems created by conflicting uses never were fully resolved (Skovlin 1984). More recently, integrated research strategies on riparian zone management have interpreted the status of existing systems and promoted management approaches aimed at enhancing or restoring the health of these systems.

This research project studied foliar cover responses of montane riparian vegetation, with respect to 4 grazing treatments. Treatment areas included vegetation protected from livestock grazing for 30 years. It was hypothesized that differences in percent relative foliar cover of key plant species and life forms would not be significant among treatments. Determination of plant community composition from foliar cover over 2 growing seasons resulted in a baseline floristic inventory, comparisons of plant community status, and short-time trend information required for future research on community status at the study area. Also, this study presents a workable method for determining floristic inventories and community status at other riparian sites in the Rocky Mountain region.

\section{Study Area}

The Sheep Creek allotment comprises an area of 5,342 ha with 1,052 ha as primary grazeable range. Lodgepole pine (Pinus contorta Dougl.) dominates the nongrazeable remainder. The allotment is located in northcentral Colorado within Roosevelt National Forest, approximately $75 \mathrm{~km}$ northwest of Fort Collins. The study was conducted at a research site within the allotment, along $5 \mathrm{~km}$ of gentle-sloping meadows adjacent to Sheep Creek. Elevation at the site is approximately $2,500 \mathrm{~m}$.

The allotment supported 1,175 animal unit months (AUMs) in the early 1900 's. Stocking rates were substantially decreased to 897 AUMs in 1949, and 740 AUMs in 1962. Current carrying capacity was estimated by the U.S. Forest Service to be 621 AUMs. A study conducted in 1985 and 1986 at this research site estimated forage utilization within riparian meadows adjacent to the creek at $65 \%$ of the current year's growth (Schulz and Leininger 1990).

The Union Pacific Railroad Company (UPRC) owns sections of uplands and riparian meadows at Sheep Creek, interspersed with sections of public land administered by the U.S. Forest Service (Fig. 1). Currently, Union Pacific Railroad Company lands are leased to permittees under a season-long grazing system. Cow-calf pairs are stocked around 20 June and are removed in late September. Livestock of Union Pacific Railroad Company permittees are free to roam on U.S. Forest Service properties.

According to U.S. Forest Service documents, all treatment areas of this study had been overgrazed from the 1890 's to 1956 . Between 1956 and 1959, the U.S. Forest Service constructed 3 exclosures by fencing $2.5 \mathrm{~km}$ of stream and adjacent riparian meadow for a total of $\mathbf{4 0}$ ha. Protected riparian areas were dedicated to fishery habitat improvement. Dense willow (Salix spp.) stands now stabilize 
Roosevelt National Forest T $11 \mathrm{~N}$

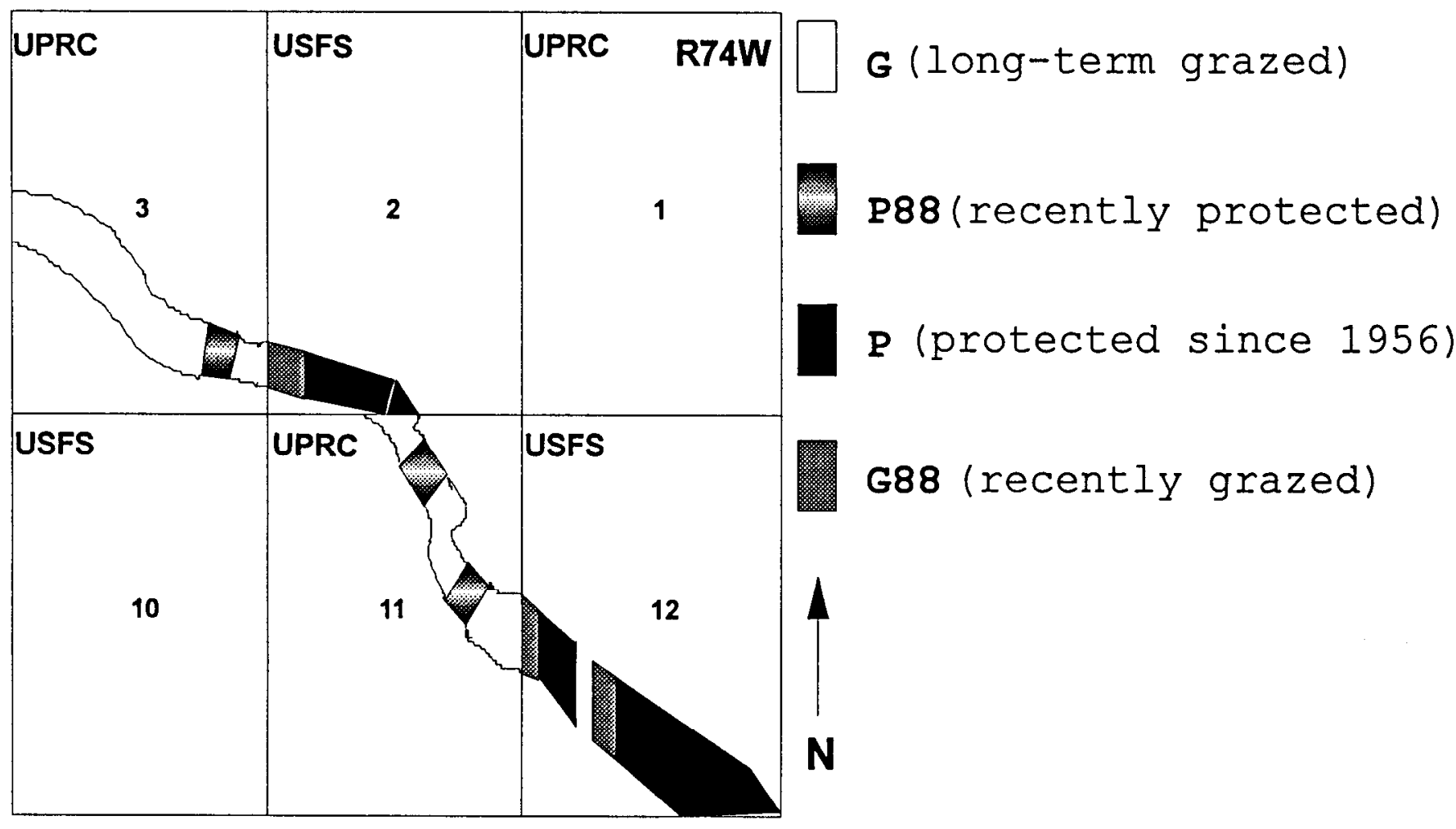

Fig. 1. Riparian corridor of the Sheep Creek, Colorado, study area showing treatments, replications, and ownership.

streambanks and shade the aquatic environment within these exclosures (Schulz and Leininger 1990). Upland areas were not protected from livestock grazing.

Three new exclosures were built in grazed areas owned by the Union Pacific Railroad Company in June and July of 1988. From upper to lower stream, these exclosures encompassed areas of 0.52 ha, 0.27 ha, and 0.48 ha. At the same time, portions of 3 old exclosures built by the U.S. Forest Service in 1956 were opened to livestock grazing at utilization levels mentioned previously. These areas were 0.29 ha, $0.68 \mathrm{ha}$, and 0.78 ha, respectively.

Carex species, particularly beaked sedge [Carex rostrata Stokes] and ovalhead sedge [Carex festivella Mack.], along with fowl bluegrass [ Poa palustris L.], were key species of those sites characterized by water seepage from adjacent uplands onto riparian meadows. Native species such as slender wheatgrass [Agropyron trachycaulum (Link) Malte.], Parry oatgrass [Danthonia parryi Scribn.], and shrubby cinquefoil [Potentilla fruticosa L.] were found exclusively in protected areas. Hay-like sedge (Carex foenea Willd.) and Baltic rush [Juncus balticus var. montanus Engelm.] were common on microsites with deep litter accumulations. Conversely, pussytoes [Antennaria parvifolia Nutt. and $A$. rosea Greene], Kentucky bluegrass [Poa pratensis L.], and common dandelion [Taraxacum officinale Wiggers] were common species of grazed sites with shallow soils. White Dutch clover [Trifolium repens L.] increased over Antennaria spp. in grazed mesic sites.

The year 1988 was characterized by a relatively wet winter and a very dry growing season, with very negligible precipitation in August $(5.8 \mathrm{~mm})$, the lowest amount on record for the month since 1948. Conversely, 1989 was characterized by low winter snow accumulation and above average summer precipitation. Three, 6 , and 4 precipitation events totalled $52.3 \mathrm{~mm}$ in June, $109.7 \mathrm{~mm}$ in July, and $53.1 \mathrm{~mm}$ in August, respectively.

\section{Methods}

Long-term grazing $(\mathrm{G})$, protection from livestock grazing since $1956(\mathrm{P})$, recent protection following long-term grazing (P88), and recent livestock grazing following protection (G88) were 4 treatments compared in this study. Three replicated areas were provided for each treatment (Fig. 1). Three 20 -m permanent transects were randomly located within each replication area. Ten $20 \times$ $50-\mathrm{cm}$ rectangular plots were randomly and permanently assigned to each transect. Percent foliar cover, as defined by the Society for Range Management (1983), was measured within these rectangular plots. Determinations were made in early August 1988, late June 1989, and early August 1989.

Data were collected by subdividing the $20 \times 50-\mathrm{cm}$ rectangular plot into 10 cover classes (USDI, Bureau of Land Management, 1985). Bare ground, litter, and individual plant species were visualized in the class that would contain their projected cover. The midrange value of each class was assigned to each observation within each plot. This method is a modification of the method developed by Daubenmire, which implements 6 cover classes (Daubenmire 1959).

Empty spaces of foliage were excluded from measurements. All species found in plots were recorded. Measurements were taken on foliar cover projected into plots by plant species growing outside plots. Trees were measured only as seedlings. Species that were present, but not visible in the veritical stratification of foliage, were assigned a first cover class midrange value. Willows were measured by Schulz and Leininger (1990) and by other co-researchers at Sheep Creek and were excluded from this study.

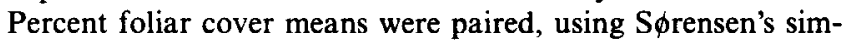
ilarity index, to determine percent similarity in foliar cover between treatments (S申rensen 1948). A distinct advantage of this 
approach is that all species encompassed by plots are indexed.

Major plant species in the Sheep Creek area are yarrow [Achillea lanulosa Nutt.]; Carex festivella, Nebraska sedge [C. nebraskensis Dewey], $C$. rostrata; tufted hairgrass [Deschampsia caespitosa (L.) Beauv.]; strawberry [Fragaria ovalis Lehm.) Rybd.]; Juncus balticus var. montanus; Poa palustris, P. pratensis; Potentilla fruticosa, herbaceous cinquefoil species [namely $P$. diversifolia Lehm., P. gracilis Dougl. ex Hook. var. pulcherrima, Lehm., $P$. hippiana Lehm., and $P$. pennsylvanica L.]; Taraxacum officinale; and Trifolium repens. Percent foliar cover data of these species were analyzed with a 2-stage nested analysis of variance (ANOVA) to determine treatment differences. Three replications were nested within each treatment and 3 transects were nested within each replication and treatment combinations. Individual measurements were nested within transects.

Replication within treatment means square was chosen as the appropriate error term for testing the significance of treatment differences. This procedure simulated an experiment where treatments and replications were randomly located, even though such random selection was not possible. This choice of error term reflected the difference among treatment means in the population of similar locations being tested.

All plant species with very sparse distributions were not analyzed statistically. However, forbs, grasses, legumes, mosses, rushes, sedges, and shrubs were analyzed as cumulative percent foliar cover of plant species belonging to each of these given life forms.

The least significant difference (LSD) method was applied to individual species foliar cover means, litter, bare ground, and life forms when F-tests were significant at the 0.05 level. This procedure identified treatments that were significantly different from each other. When F-tests were not significant, LSD values were viewed as indicative of accuracy, but were not used to declare significant differences due to their additive error rate per comparison (Steel and Torrie 1980). However, some least significant differences were carried out on foliar cover comparisons with $p$ values $\leq 0.10$ as well, in order to get indications of treatment effects on foliar cover of common species and life forms. All statistical tests used type I error rate of $\alpha=0.05$.

Steel and Torrie (1980) recommended using square root transformations on percentage data with a range between 0 and $20 \%$ to make means and variances independent and variances stable. Square root transformations were performed on foliar cover data, with the exception of litter cover, before proceeding with ANOVA and LSD tests.

\section{Results}

S $\phi$ rensen's similarity indices for treatment comparisons of community status are presented in Table 1. Long-term grazing and

\begin{abstract}
Table 1. Similarity indices based on percent foliar cover estimates of vegetation found within treatments from the Sheep Creek, Colorado, study area. Values range from 0 to $100 \%$.
\end{abstract}

\begin{tabular}{lccc}
\hline \hline $\begin{array}{l}\text { Treatment } \\
\text { comparison }\end{array}$ & Aug. 1988 & Jun. 1989 & Aug 1989 \\
\hline G-P88 & 71.18 & 65.45 & 63.64 \\
G-P & 55.53 & 55.62 & 57.06 \\
G-G88 & 47.23 & 57.81 & 56.95 \\
P88-P & 56.08 & 69.41 & 66.98 \\
P88-G88 & 47.84 & 67.02 & 63.01 \\
P-G88 & 77.37 & 84.36 & 82.01 \\
\hline
\end{tabular}

G-P88 (long-term grazing-recent protection)

G-P (long-term grazing-long-term protection)

G-G88 (long-term grazing-recent grazing)

P88-P (recent protection-long - term protection)

P88-P (long-term protection-recent grazing)

P-G88 (protected-recent grazed) recent protection following long-term grazing decreased in similarity from 1988 to 1989 , following short-term grazing exclusion in the latter. Long-term grazing/recent livestock grazing following protection, long-term protection/recent livestock grazing following protection, and recent protection following long-term grazing/long-term protection showed an increase in similarity from 1988 to 1989 . Recent protection following long-term grazing/recent livestock grazing following protection, the short-term reverses of historical treatments, resulted in the highest increase in percent similarity among all comparisons. Long-term grazing/long-term protection treatments appeared to have stable, but low, similarity over the 2-year study.

Foliar cover data of plant life forms are presented in Table 2. Percent foliar cover of grasses was significantly different among treatments in 1988 at the 0.10 level $(P=0.078)$. Conversely, similarities were found in June $1989(P>0.38)$ and August $1989(P>0.19)$. It was determined that grass cover was greater in recent protection following long-term grazing than in long-term protection and recent livestock grazing following protection (Table 2).

Cover of legumes was significantly different among treatments in August 1988, June 1989, and August (1989) $(P=0.02)$. It was determined by least significant difference analysis that recent protection following long-term grazing was significantly different from long-term grazing, but similar to long-term protection and recent livestock grazing following protection by August 1989 (Table 2). Foliar cover of Trifolium repens within recent protection following long-term grazing decreased dramatically from 1988 to 1989 (Table 3).

There were no significant differences among treatments for foliar cover of forbs $(P>0.69)$, mosses $(P>0.23)$, rushes $(P>0.18)$, and shrubs $(P>0.25)$. Percent foliar cover of sedges was not significantly different among treatments $(P>0.88)$. Carex festivella appeared to be most affected by treatments $(P>0.10$ in August 1988, $P>0.057$ in June 1989 , and $P>0.12$ in August 1989) than other sedges (Table 3). Carex nebraskensis $(P>0.34)$ and $C$. rostrata $(P>0.85)$ were unaffected by treatments.

There were significant treatment differences for bare ground at the 0.05 and 0.10 levels ( $P=0.01$ in August 1988, $P=0.10$ in June 1989 , and $P=0.051$ in August 1989). It was determined by least significant differences analysis that the long-term grazing treatment was significantly different from all other treatments in August 1988, but increased similarities with other treatments were obtained in 1989 (Table 3).

Litter cover was significantly different in 1988 at the 0.10 level only $(P=0.064)$. Protected areas had higher litter accumulations than any other treatment areas by 1989 (Table 3).

Foliar cover of Taraxacum officinale was significantly different in June $1989(P=0.03)$ (Table 3). Dandelion densities were 3 times greater in long-term grazed areas (Popolizio 1990). However, individual plants in these areas displayed stunted growth under grazing pressure and trampling. This may explain similarities among treatments late in the season ( $P>0.19$ in August 1988 and $P>0.11$ in August 1989).

Foliar cover of Trifolium repens was significantly different in August $1988(P=0.04)$, June $1989(P=0.03)$ and August $1989(P=$ 0.03 ) (Table 3). Results were consistent with those presented for legumes grouped as life forms (Table 2). Alpine milkvetch [Astragalus alpinus L.], showy locoweed [Oxytropis splendens Dougl.], golden pea [Thermopsis divaricarpa Nels.], and red clover [Trifolium pratense L.], were recorded only infrequently.

Foliar cover of Achillea lanulosa $(P>0.69)$, Deschampsia caespitosa $(P>0.16)$, Fragaria ovalis $(P>0.43)$, Juncus balticus var. montanus ( $P>0.19)$, Poa palustris $(P>0.45)$, Poapratensis $(P>0.13)$, Potentilla fruticosa $(P>0.22)$, and herbaceous Potentilla spp. ( $P>0.58$ ) was not significantly different among treatments (Table 3). 
Table 2. Mean percent foliar cover of life forms within treatments from the Sheep Creek, Colorado, study area. Data from August 1988, June 1989, and August 1989.

\begin{tabular}{|c|c|c|c|c|c|c|c|c|c|c|c|c|}
\hline \multirow[b]{2}{*}{ Treatment } & \multicolumn{4}{|c|}{ Aug. 1988} & \multicolumn{4}{|c|}{ Jun. 1989} & \multicolumn{4}{|c|}{ Aug. 1989} \\
\hline & G & P88 & $\mathbf{P}$ & G88 & G & P88 & $\mathbf{P}$ & G88 & G & P88 & $\mathbf{P}$ & G88 \\
\hline & & - & $\theta-$ & $\ldots$ & - & $\ldots$ & . & - - - & $\cdots$ & $\cdots$ & $\ldots$ & $\cdots$ \\
\hline Legumes ${ }^{2}$ & 12.93 & 9.45 & 0.17 & $0.28^{*}$ & 15.61 & 3.11 & 0.42 & $0.78^{*}$ & 14.25 & 2.04 & 0.62 & $0.94^{*}$ \\
\hline Mosses & 2.99 & 0.89 & 0.18 & 3.24 & 1.60 & 0.21 & 0.21 & 1.81 & 2.18 & 0.47 & 0.32 & 1.46 \\
\hline Rushes & 1.17 & 2.10 & 3.10 & 2.45 & 0.93 & 1.78 & 2.04 & 2.03 & 1.10 & 1.97 & 2.28 & 2.01 \\
\hline
\end{tabular}

- Differences among treatments for a given sampling period were significant at the 0.10 level.

* Differences among treatments for a given sampling period were significant at the 0.05 level.

I August 1988: $P 88 \approx P$ and $G 88(P=0.078)$.

2August 1988: $G=P 88 \approx G 88=P$; June 1989: $G \approx P$ and G88; August 1989: $G \sim P 88=G 88=P$.

Willows were not measured.

$\mathbf{G}=$ long-term grazing

P88 = recently protected following long-term grazing.

$P=$ protected from grazing since 1956

$\mathrm{G} 88$ = recently grazed following protection.

Table 3. Mean percent foliar cover of litter, bare ground, and individual plant species within treatments from the Sheep Creek, Colorado, study area. Data from August 1988, June 1989, and August 1989.

\begin{tabular}{|c|c|c|c|c|c|c|c|c|c|c|c|c|}
\hline \multirow[b]{2}{*}{$\begin{array}{l}\text { No. of Species } \\
\text { Treatment }\end{array}$} & \multicolumn{4}{|c|}{ Aug. 1988} & \multicolumn{4}{|c|}{ Jun. 1989} & \multicolumn{4}{|c|}{ Aug. 1989} \\
\hline & $\begin{array}{l}50 \\
G\end{array}$ & $\begin{array}{c}52 \\
\mathbf{P} 88\end{array}$ & $\begin{array}{l}53 \\
P\end{array}$ & $\begin{array}{c}46 \\
\text { G88 }\end{array}$ & $\begin{array}{l}50 \\
G\end{array}$ & $\begin{array}{c}55 \\
\text { P88 }\end{array}$ & $\begin{array}{l}60 \\
\mathbf{P}\end{array}$ & $\begin{array}{c}55 \\
\text { G88 }\end{array}$ & $\begin{array}{l}50 \\
\mathrm{G}\end{array}$ & $\begin{array}{c}53 \\
\text { P88 }\end{array}$ & $\begin{array}{l}56 \\
P\end{array}$ & $\begin{array}{c}54 \\
\text { G88 }\end{array}$ \\
\hline & \multicolumn{4}{|c|}{$\ldots \ldots+\ldots$} & \multicolumn{4}{|c|}{$\ldots \ldots+\ldots-\ldots, \ldots$} & \multicolumn{4}{|c|}{$\ldots \ldots \ldots \ldots$} \\
\hline Achillea lanulosa & 2.16 & 2.19 & 1.43 & 1.06 & 2.81 & 2.75 & 1.83 & 1.79 & 2.89 & 2.96 & 1.72 & 1.96 \\
\hline Agoseris glauca & 0.03 & - & 0.46 & 0.03 & 0.03 & 0.03 & 0.03 & 0.03 & 0.03 & 0.03 & 0.39 & 0.03 \\
\hline Agropyron trachycculum & - & 0.03 & 0.53 & 0.18 & - & 0.21 & 0.67 & 0.22 & - & 0.21 & 0.74 & 0.29 \\
\hline Agrostis scabra & 0.06 & 0.35 & 0.14 & 0.06 & 0.17 & 0.31 & 0.25 & 0.11 & 0.25 & 0.36 & 0.25 & 0.19 \\
\hline Agrostis stolonifera & 1.69 & 0.39 & 0.46 & 0.40 & 0.97 & 0.25 & 0.18 & 0.56 & 1.68 & 0.25 & 0.25 & 0.78 \\
\hline Allium geyeri & - & 0.03 & - & - & $\longrightarrow$ & - & - & 0.14 & - & 一 & - & 0.06 \\
\hline Alopecurus pratensis & & $\ldots$ &.- & 0.71 & $\ldots$ & $\ldots-$ & 0.03 & 1.75 & - & -- & 0.03 & 1.29 \\
\hline Antennaria spp. & 0.68 & 0.67 & 0.43 & 0.49 & 0.74 & 0.61 & 0.46 & 0.44 & 0.90 & 0.67 & 0.51 & 0.65 \\
\hline Arnica chamissonis & - & 0.11 & 0.08 & 0.19 & $\ldots$ & 0.61 & 0.22 & 0.46 & - - & 0.14 & 0.33 & 0.33 \\
\hline Arnica fulgens & - & - & 0.22 & - & - & -- & 0.22 & - & - - & $\ldots$ & 0.29 & - - \\
\hline Artemisia frigida & 0.06 & 0.03 & - & - & 0.06 & 0.03 & - & - & 0.06 & 0.03 & - & - \\
\hline Artemisia tridentata & 0.10 & - & - & - & 0.21 & - & - & - & 0.21 & - & - & - \\
\hline Aster hesperius & 0.19 & 0.31 & 0.82 & 0.60 & 0.24 & 0.31 & 1.12 & 0.96 & 0.19 & 0.37 & 1.35 & 1.12 \\
\hline Astragalus alpinus & 0.26 & 0.31 & $\ldots$ & 0.06 & 0.26 & 0.42 & 0.11 & 0.08 & 0.33 & 0.42 & 0.11 & 0.08 \\
\hline Bare ground & 15.39 & 2.96 & 3.06 & $3.46^{*}$ & 7.40 & 1.92 & 2.76 & $4.72^{\circ}$ & 7.89 & 1.26 & 2.44 & $5.62^{\circ}$ \\
\hline Caltha leptosepala & 0.06 & 0.15 & - & - & 0.12 & 0.29 & - & - & 0.19 & 0.22 & - & - \\
\hline Campanula parryi & - & 0.08 & - & - & - & 0.03 & 0.11 & $\ldots$ & - & 0.06 & 0.11 & $\ldots$ \\
\hline Campanula rotundifolia & - & 0.11 & 0.03 & - & - & 0.06 & - & - & - & 0.06 & $\underline{-11}$ & 0.03 \\
\hline Carex aquatilis & - & - & - & 0.06 & - & - & - & 0.12 & - & $\ldots$ & - & 0.12 \\
\hline Carex athrostachya & 0.94 & 0.85 & 0.47 & 0.15 & 1.36 & 0.62 & 0.40 & 0.08 & 1.18 & 0.76 & 0.47 & 0.08 \\
\hline Carex festivella ${ }^{1,3}$ & 2.42 & 4.15 & 2.31 & $0.92^{\circ}$ & 3.06 & 2.53 & 1.94 & $1.42^{\circ}$ & 3.08 & 3.89 & 2.08 & 1.14 \\
\hline Carex foenea & 0.18 & 0.38 & 1.76 & 0.31 & 0.29 & 2.19 & 1.08 & 0.33 & 0.18 & 2.36 & 1.54 & 0.26 \\
\hline Carex microptera & 0.40 & 0.15 & 0.62 & - & 0.68 & 0.08 & 0.44 & - & 0.40 & 0.22 & 0.53 & $\ldots$ \\
\hline Carex nebraskensis ${ }^{1}$ & 1.25 & 6.32 & 2.61 & 1.64 & 1.94 & 3.96 & 2.15 & 2.24 & 2.08 & 4.92 & 2.64 & 2.76 \\
\hline Carex praticola & - - & 0.44 & 1.06 & 1.86 & - & 0.47 & 0.87 & 1.50 & - & 0.79 & 1.06 & 1.71 \\
\hline Carex rostratal & 8.32 & 4.90 & 8.79 & 5.06 & 7.25 & 2.40 & 7.24 & 7.44 & 8.37 & 3.01 & 9.07 & 7.25 \\
\hline Other Carex spp. & - & 0.06 & - & 1.57 & - & 0.22 & - & 1.40 & - & 0.15 & - & 1.53 \\
\hline Cerastium spp. & 0.31 & 0.03 & - - & - - & 0.25 & 0.08 & 0.06 & 0.06 & 0.22 & 0.03 & 0.06 & 0.03 \\
\hline Cirsium flodmanii & 0.28 & 0.12 & 0.03 & 0.32 & 0.44 & 0.35 & 0.03 & 0.28 & 0.54 & 0.37 & 0.06 & 0.60 \\
\hline Danthonia parryi & 0.03 & - & 0.64 & 1.61 & 0.03 & - - & 0.50 & 2.15 & 0.03 & - - & 0.92 & 2.15 \\
\hline Deschampsia caespitosal & 0.76 & 4.44 & 0.78 & 1.22 & 0.71 & 2.93 & 0.76 & 0.54 & 0.76 & 5.72 & 0.89 & 1.36 \\
\hline Eleocharis pauciflora & 0.33 & 0.26 & 0.06 & 0.83 & 0.36 & 0.24 & 0.06 & 0.06 & 0.36 & 0.24 & 0.06 & 0.08 \\
\hline Epilobium paniculatum & 0.06 & - & - & 0.03 & 0.03 & - & -- & 0.03 & 0.10 & - & $\ldots$ & 0.11 \\
\hline Equisetum arvense & 0.62 & 0.14 & 0.42 & 0.03 & 0.83 & - - & 0.36 & 0.22 & 0.50 & - & 0.42 & 0.17 \\
\hline Erigeron spp. & - & 0.36 & 0.58 & 0.25 & - & 0.15 & 0.93 & 0.53 & 0.06 & 0.26 & 1.06 & 0.57 \\
\hline Fragaria ovalis ${ }^{l}$ & 0.24 & 一 & 1.94 & 3.01 & 0.28 & - & 1.44 & 2.99 & 0.37 & 0.03 & 1.71 & 3.44 \\
\hline Galium boreale & - & - & 0.06 & 0.31 & -2 & - & 0.12 & 0.25 & - & - & 0.15 & 0.43 \\
\hline Galium trifidum & - & - & 0.05 & - & - & - & 0.08 & - & - & - & 0.08 & - \\
\hline Gentianella amarella & $\overline{-1}$ & 0.11 & - & - & - & 0.06 & -- & - & - & 0.06 & - & - \\
\hline Geum macrophyllum & 0.32 & - & 0.11 & 0.11 & 0.29 & - & 0.03 & 0.11 & 0.44 & - & 0.12 & 0.11 \\
\hline Geum trifolium & - - & - & 0.06 & - & - & - & 0.06 & - - & - - & - & 0.08 & - \\
\hline Glyceria striata & 0.11 & - & - - & -- & 0.14 & 0.03 & - & $-\cdots$ & 0.14 & 0.03 & - & -- \\
\hline Hippochaete variegata & $-\overline{c o s}$ & - & $\overline{0}$ & - & - & - & 0.06 & - & - - & - & 0.06 & - - \\
\hline Hordeum spp. & 0.10 & 0.06 & 0.61 & - & 0.03 & 0.08 & 0.18 & 0.03 & 0.10 & 0.08 & 0.43 & 0.06 \\
\hline
\end{tabular}


Table 3. Continued.

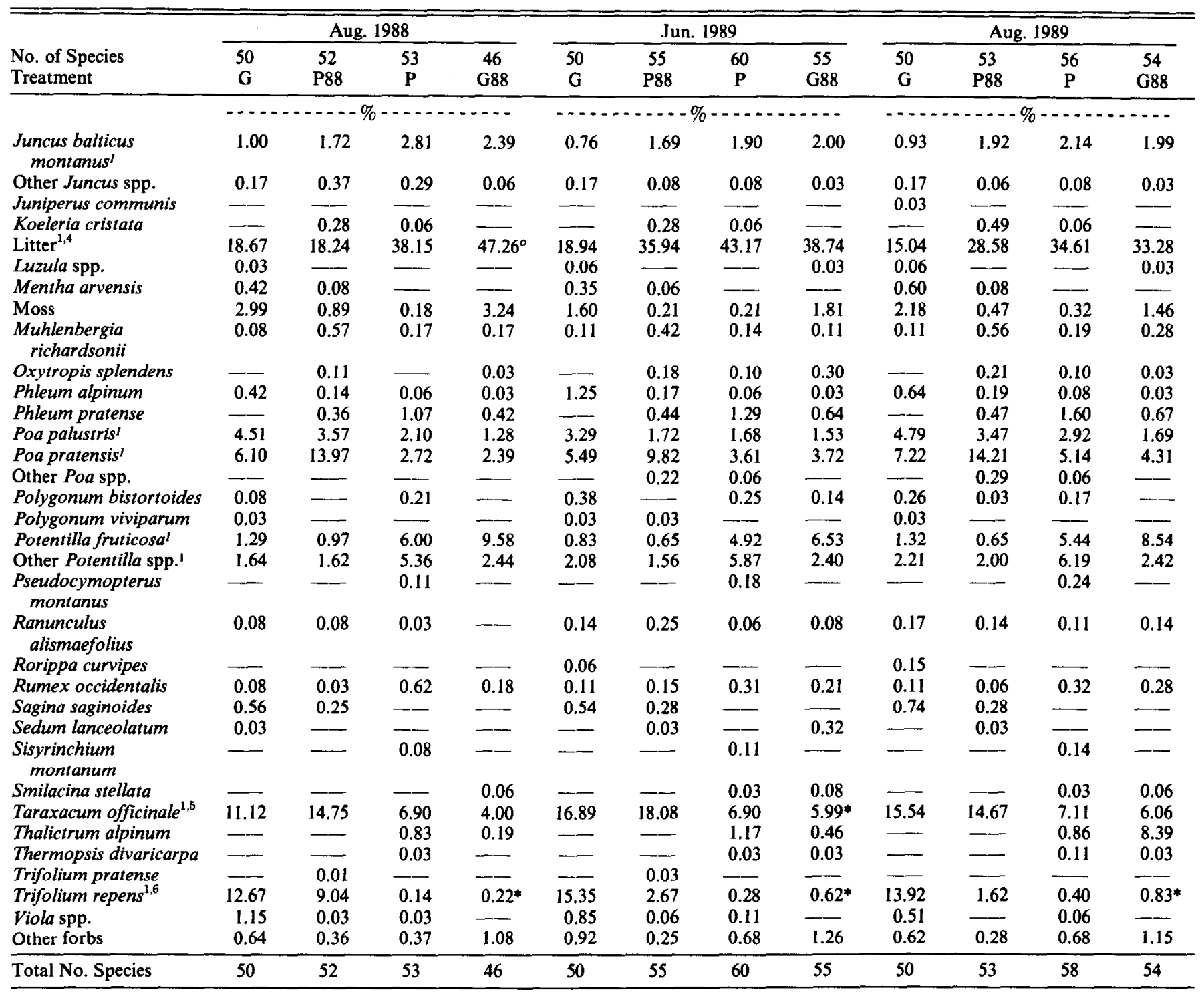

1Analyzed statistically.

-Differences among treatments for a given sampling period were significant at the 0.10 level.

* Differences among treatments for a given sampling period were significant at the 0.05 level.

2August 1988: $G \approx P 88=P=G 88 ;$ June $1989 ; G \approx P 88(P=0.10)$; August $1989: G=G 88, G 88=P=P 88$.

${ }^{3}$ August 1988: $P 88 \approx G 88(P=0.10)$; June 1989: $G$ and P88 G88 ( $\left.P=0.057\right)$.

4August 1988: $G 88 \approx G$ and $P 88(P=0.064)$.

sJune 1989: $P 88=G \approx P=G 88$

6August 1988: $G \approx G 88$ and $P$; June 1989: $G \approx G 88$ and $P$; August 1989: $G \approx P 88=G 88=P$.

$G=$ long-term grazing

P88 = recently protected following long-term grazing.

$P=$ protected from grazing since 1956

$G 88=$ recently grazed following protection.

\section{Discussions and Conclusions}

Significant differences of percent cover means may have resulted from excessive forage utilization in grazed areas and heavy litter build-up in exclosures. Bare ground, legumes, particularly Trifolium repens, and Taraxacum officinale were found to be significantly higher in long-term grazed treatments. Bare ground may have decreased significantly in this treatment from 1988 to 1989 as a result of a very moderate grazing season and high precipitation in 1989.

The inhibiting effect of litter accumulation on the foliar cover of small forbs and legumes may have resulted in an observed 5-fold decrease of Trifolium repens from 1988 to 1989 in recently built exclosures (recent protection following long-term grazing). Litter cover increased $10 \%$ in this treatment.

Percent foliar cover of forbs was not significantly different among treatments. Forb composition in grazed areas was dominated by Taraxacum officinale. A greater proportion of native species were found to be growing within old exclosures than in open grazed areas.

Individual sedge spp. responded differently to treatments. Carex nebraskensis is highly preferred as forage by livestock (Winward 1986); this sedge species may withstand heavy utilization as a result of its rhizomatous habit and location in habitats with favorable soil moisture conditions (USDA Forest Service 1937). Cattle utilize 
Carex rostrata early in the growing season, but use decreases as shoots become coarse (Hansen et al. 1988). C. rostrata stands received little disturbance during the 2-year study. Swollen spike sedge ( Carex athrostachya Olney) was most common in long-term grazed areas, intermingling with Carex festivella. Carex foenea and meadow sedge ( $C$. praticola $\mathrm{Rydb}$.) were found predominantly in long-term protected areas.

In a previous study at Sheep Creek, Schulz and Leininger (1990) reported significant differences between mean percentage canopy cover of bare ground, Poa palustris, $P$. pratensis, litter, Trifolium repens, and grasses and shrubs as life forms in long-term grazed and protected areas $(P=0.05)$. Forb cover was not significantly different between these 2 treatments. They reported that the average percent canopy cover of Salix spp. was $17 \%$ in exclosures versus $2 \%$ in grazed areas $(P>0.004)$. An arcsin square root transformation was applied to cover data prior to their analyses. Shortterm grazing and protection had not been established at the time of their study.

Two years of grazing in long-term protected areas appeared to have a stimulating effect on plant foliar cover; however, impacts on other environmental characteristics of the system should be monitored by future studies. Concurrently, 2 years of protection in long-term grazed areas resulted in increased litter accumulation, decreased bare ground, and increased foliar cover of forage species (e.g., grasses and sedges) when compared to long-term grazed areas.

Past heavy grazing in the Sheep Creek area has resulted in changes in plant community composition. Indeed, the recent return of beaver in protected portions of Sheep Creek resulted in visual aspect dominance of water-loving plant species. Differences resulting from grazing and protective treatments became more pronounced when less frequent and rare species were considered (Popolizio 1990).

This study of percent foliar cover alone cannot assess merits and drawbacks of grazing strategies and protective measures, but must rather be incorporated as one of many studies in evaluating and understanding complex relationships of riparian systems. Identification of soil types, soil porosity and depth, soil organic matter content, litter deposition, channel morphology, streambank erosion, sediment load, willow cover, forage production, percent utilization, plant density, and plant frequency, along with foliar cover, would be most useful parameters of study in understanding riparian systems' responses, particularly those relating to grazing strategies and protective measures. Internal and continuous grazing pressures in riparian areas appear to have more detrimental impacts on soil properties than on a plant community (Winward 1986).

The $20 \times 50$-cm rectangular plot proved to be an inappropriate technique for deriving reliable estimates of shrub foliar cover. In future studies, direct or indirect density techniques may be more appropriate measures of shrub abundance within treatment areas. Shrub foliar cover may be measured more accurately as a line intercept for each random transect.

Concurrent application of the inclined point method with the 20 $\times 50-\mathrm{cm}$ rectangular plot would alleviate bias in estimating foliar cover of narrow leaf or leafless species such as Juncus balticus var. montanus. Floyd and Anderson (1987) compared the precision of line interception, point interception, and quadrat cover estimates of sagebrush steppe vegetation. Similar studies comparing methods would be useful in determining the precision, workability, and most appropriate use of plots in riparian studies.

Finally, depth of litter accumulations as an $O$ horizon may be more meaningful measures than surface litter cover readings in plots. Volland (1978) reported significant differences in litter depth between grazed and rested Poa pratensis meadows over an 11-year period.

\section{Literature Cited}

Bohn, C.C., and J.C. Buckhouse. 1985. Some responses of riparian soils to grazing management in northeastern Oregon. J. Range Manage. 38:378-381.

Daubenmire, R. 1959. A canopy-coverage method of vegetation analysis. Northwest Sci. 33:43-64.

Dyksterhuis, E.J. 1949. Condition and management of rangeland based on quantitative ecology. J. Range Manage. 2: 104-115.

Floyd, D.A., and J.E. Anderson. 1987. A comparison of three methods for estimating plant cover. J. Ecol. 75:221-228.

Hansen, P.L., S.W. Chadde, and R.O. Pnster. 1988. Riparian dominance types of Montana. Misc. Pub. 49, Montana Forest and Conserv. Exp. Sta., School of Forestry, Univ. of Montana, Missoula.

Knopf, F.L., R.R. Johnson, T. Rich, F.B. Samson, and R.T. Szaro. 1988. Conservation of riparian ecosystems in the United States. Wilson Bull. 100:272-284.

Laycock, W.A., and P.W. Conrad. 1967. Effect of grazing on soil compaction as measured by bulk density on a high elevation cattle range. $J$. Range Manage. 20:136-140.

Orr, H.K. 1960. Soil porosity and bulk density on grazed and protected Kentucky bluegrass range in the Black Hills. J. Range Manage. 13:80-86.

Platts, W.S. 1981. Streamside management to protect bank-channel stability and aquatic life. p. 245-256. In: D.M. Baumgartner (ed.) Interior West Watershed Management, Washington State Univ., Coop. Ext., Pullman.

Platts, W.S., and R.F. Raleigh. 1984. Impacts of grazing on wetlands and riparian habitat. p. 1105-1117. In: Developing strategies for range management, Westview Press, Boulder, Colo.

Platts, W.S., and R.L. Nelson. 1985. Streamside and upland vegetation use by cattle. Rangelands 7:5-7.

Popolizio, C.A. 1990. Successional status of montane riparian vegetation under four levels of grazing. M.S. Thesis, Colorado State Univ.

Skinner, Q. 1986. Riparian zones then and now. p. 8-22. In: Proc. Wyoming Water 1986 and Streamside Zone Conf. Univ. Wyoming, Laramie.

Skovlin, J.M. 1984 . Impacts of grazing on wetlands and riparian habitat: a review of our knowledge. p. 1001-1103. In: Developing strategies for range management. Westview Press, Boulder, Colo.

Society for Range Management. 1983. Guidelines and terminology for range inventories and monitoring. Rep. of Range Inventory Standardization Committee.

S $\phi$ rensen. T. 1948. A method of establishing groups of equal amplitude in plant sociology based on similarity of species content. Biol. Skr. 5:1-34.

Steel, R.G.D and James H. Torrie. 1980. Principles and procedures of statistics: a biometrical approach. McGraw-Hill Book Co. 633 p.

Schulz, T.L.T., and W.C. Leininger. 1990. Differences in riparian vegetation structure between grazed areas and exclosures. J. Range Manage. 43:294-298.

USDA Forest Service. 1937. Range plant handbook. U.S. Gov. Print. Off., Washington, D.C.

USDI Bureau of Land Management. 1985. Rangeland monitoring. Trend studies. Superintendent of Documents. U.S. Gov. Print. Off., Washington, D.C. TR4404-4.

Van Velson, R. 1979. Effects of livestock grazing upon rainbow trout in Otter Creek, Nebraska. P. 53-55. In: O.B. Cope (ed.) Proc. ForumGrazing and Riparian/Stream Ecosystems, Trout Unlimited, Inc., Denver, Colo.

Volland, L.A. 1978. Trends in standing crop and species composition of a rested Kentucky bluegrass meadow over an 11-year period. p. 525-529. In: D.N. Hyder (ed.) Proc. 1st Int. Range Congr., Denver, Colo.

Winward, A.H. 1986. Vegetation characteristics of riparian areas. Trans. Western Section of the Wildl. Soc. 22:98-101. 